\title{
Wireless sensor network node optimal coverage based on improved genetic algorithm and binary ant colony algorithm
}

\author{
Jingwen Tian*, Meijuan Gao and Guangshuang Ge
}

\begin{abstract}
Considering the situation of some practical factors such as energy saving of the nodes and the high density of distributing nodes in wireless sensor networks, a wireless sensor network (WSN) node optimal coverage method based on improved genetic algorithm and binary ant colony algorithm is proposed in this paper. The genetic algorithm and ant colony algorithm are improved and fused aiming at their disadvantages. The binary code expects a low intelligence of each ant, and each path corresponds to a comparatively small storage space, thus considerably improving the efficiency of computation. The optimal working node set is computed according to the max-coverage area of working sensor and the min-number of working sensor constraint conditions to optimize algorithm. The simulation results demonstrate that the proposed algorithm can converge at the optimal solution fast and satisfy the requirement of low node utilization rate and a high coverage rate, thus prolonging the network lifetime efficiently.
\end{abstract}

Keywords: Wireless sensor network, Node optimal coverage, Genetic algorithm, Binary ant colony algorithm

\section{Introduction}

A wireless sensor network is made up of a large number of sensor nodes which possess perceptual ability, calculation of capacity, and communication ability with self-organizing style. Sensor nodes complete the data collection and transmission together. It has wide application such as battlefield surveillance, environmental protection, intelligent home furnishing, and so on [1-6]. However, sensor nodes usually adopt limited-capacity battery to provide energy due to the limit of volume and cost. The energy cannot regenerate or supply. So the coverage and energy consumption are the key problems of wireless sensor network research.

At present, many scholars have done a lot of research for the node location and coverage optimization algorithm in wireless sensor network, and some feasible and effective algorithms were proposed such as Huang algorithm [7], TianD algorithm [8], CCP algorithm [9], and so on [10-12]. Chi-Fu Huang and $\mathrm{Yu}$-Chee Tseng proposed a polynomial time algorithm (polynomial-time algorithms) in the literature [4]; in the sensor network, it is a distributed protocol method, which has positioning

\footnotetext{
* Correspondence: jingwentianwcn@163.com

College of Information Technology, Beijing Union University, Beijing 100101, China
}

capabilities, environmental monitoring capability, and fault tolerant capability. Liang Ying, Zeng Peng, and Yu Haibin, in reference [7], proposed using adaptive cluster head communication (cluster-heads communication) method. The method can ensure that energy consumption is balanced in the whole network and can improve the network life cycle. Mihaela Cardei and Ding-Zhu Du, in the literature [8], proposed a rule and method to establish a "maximum disjoint set cover problem." The method is that nodes which are included in the maximum disjoint coverage area are at work, while other nodes are in a dormant state, which saves the system energy and prolongs the network life cycle. Jia Jie, Chen Jian, Chang Guiran, Zhao Linliang, and Wang Guangxing, in the literature [11], put forward the two based on weighted genetic algorithm and constraint genetic algorithm-based optimization coverage mechanism. According to generated fitness function to do genetic algorithm operation and calculate the sensor network, full coverage region is required for the approximation of the optimal set of nodes and complete working node selection, thereby reducing the redundant network and prolonging the survival time of the network. Xu et al. [13-17] proposed a crowdsourcing-based framework for processing mobile network data. Joon-Min Gil and 
Youn-Hee Han, in the literature [10], proposed a goal based on genetic algorithm coverage scheduling scheme, using evolutionary global search techniques for monitoring all targets and that can find the optimal coverage set, prolonging the network life cycle. Mohamed Aadhil M. and Roselin M.E.J., in the literature [12], using the genetic algorithm to optimize the node placement method, the method can achieve the purpose of optimizing the node coverage.

However, these algorithms have some limitations. The complexity of Huang algorithm is too high, and it could easily lead to the blockage of the channel when the communication data is large. The computing complexity and communication complexity of the TianD algorithm are lower, but there are two problems: first, inability to make the redundant nodes of monitoring area boundary dormant and second, without consideration of the overlap between node coverage areas, which led to elected excessive working nodes, thus affecting the lifetime of the network. CCP algorithm is not suitable for high-density sensor networks.

Based on the above issues and the special predominance of genetic algorithm and ant colony algorithm possess, a WSN node optimal coverage method based on improved genetic algorithm and binary ant colony algorithm is proposed in this paper, based on the location information of the nodes to build node coverage optimization model and then using the improved genetic algorithm and binary ant colony algorithm to solve the optimization model. The optimal solution is used as the working nodes of wireless sensor networks, the remaining nodes sleep to save energy. The simulation results show that this algorithm proposed in this paper effectively improves the coverage rate of the wireless sensor network.

\section{Wireless sensor network coverage optimization 2.1 Wireless sensor network coverage}

The network coverage is the basic problem of the construction of wireless sensor networks. The network coverage needs to reach the maximum under the conditions that guarantee a certain quality of service (QoS) [18-21], by measuring the network coverage to determine whether the presence of communication blind area and get the monitoring area of wireless sensor network coverage. The sensor node distribution can be adjusted or sensor nodes can be added according to the measurement condition. In addition, by changing the density of the network coverage, the more sensor nodes were deployed in some important areas in order to guarantee the reliability of measured data. Therefore, the network coverage is not only for communication coverage and monitoring area coverage but also for better meeting of the needs of certain applications. Generally speaking, quality of service is the measure of wireless sensor network coverage [22].
The wireless sensor network coverage is mainly divided into certainty coverage and the random covering. For the certainty coverage, the coverage area size is determined in order to cover the area using the minimum number of sensors. The certainty coverage is adopted to deploy the sensor nodes of a known region in this paper.

At present, a kind of widely used strategy is choosing part nodes which can be enough to cover the monitoring area as working nodes, at the same time, turn off redundant nodes [23]. A certain number of sensor nodes are deployed in the monitoring area, under the ensuring network connectivity in normal condition, letting part of the redundant nodes into low-power sleep state, while the rest of the active node is used to cover the whole region in the most likely small amount, which form the optimal coverage node set. In the wireless sensor network, the node number in the increased network not only means increased cost but also brings the limited bandwidth congestion because of the inter-node communication conflict. The optimal coverage node set is the minimum node set which does not affect the entire network coverage. This way does not only reduce potential conflicts in a wireless channel and reduce the possibility of competing for access to the media but also save energy in order to prolong the network lifetime [11].

\subsection{Wireless sensor network optimization coverage model} Supposed that the target monitoring area $A$ is a twodimensional plane, and it will be divided into $m \times n$ grid, and each grid area is 1 . The $N$ sensor nodes are randomly distributed in the $A$, the sensor node set $S$ can be expressed as $S=\left(s_{1}, s_{2}, \cdots, s_{i}, \cdots, s_{N}\right)$. The $\left(x_{i}, y_{i}\right)$ is the coordinate of sensor node $s_{i}$ in a target monitoring area $A$, each node coordinate is known, and effective perception radii are rand $s_{i}=\left\{x_{i}, y_{i}, r\right\}$. Perception range of node $s_{i}$ is a circular region with the coordinates $\left(x_{i}, y_{i}\right)$ of node $s_{i}$ as the center and the $r$ as the perception radius, to express with $c_{i}=\left\{p \in A \mid d\left(p,\left(x_{i}, y_{i}\right)\right) \leq r, \quad i \in[1, \quad N]\right\}$, where, $p$ is an arbitrary point of area $A$, and $d$ is the Euclidean distance. The perception range of the whole sensor network is $C=\cup_{i \in[1, N]} c_{i}$, that all network nodes perceptual range are in union.

There is $m \times n$ target point. $\left(a_{1}, a_{2}, \cdots, a_{j}, \cdots a_{m \times n}\right)$ need to be monitored or sensed in target monitoring area $A$; suppose $a_{j}$ is $s_{i}$ perception then $d\left(a_{j}, s_{i}\right) \leq r$; an arbitrary point of monitoring area $A$ is at least perceived by a sensor node. Assume the communication range of sensor node $s_{i}$ is a circle with the coordinates $\left(x_{i}, y_{i}\right)$ of node $s_{i}$ as the center and the $r_{c}$ as the radius; if $d\left(s_{i}, s_{j}\right) \leq$ $r_{c}$, then the sensor node $s_{i}$ and $s_{j}$ can communicate directly.

Definition 1: given a sensor node set $S$, the communication network $G=(V, E),\left\{V=S, L\left(s_{i}, s_{j}\right) \in E\right\}$ composed of $S$ is an undirected graph, if there is a communication path between any two nodes in the communication 
diagram $G$ derived from $S$ then the communication diagram is connected, when $d\left(s_{i}, s_{j}\right) \leq r_{c}$.

Definition 2: for the sensor node set $S$ and the target monitoring area $A$; if every point of $A$ is perceived by a sensor node of $S$ then the $S$ is a covering set of $A$. If the communication diagram derived from $S$ is connected then the $S$ is a connected cover set of $A$.

Definition 3: for the connected cover set $S$ and the target monitoring area $A$, the minimal connected cover set is looking for a minimal subset $S^{\prime} \quad S$, while the monitoring area $A$ is covered by $S^{\prime}$ completely and the communication diagram derived from $S^{\prime}$ is connected.

On the basis of energy efficiency, this paper presents optimization goal of a connectivity covering set: ensuring that each target point in $A$ is at least covered by a sensor node, at the same time, ensuring that a subset is a connected set.

In order to ensure connectivity, the communication radius $r_{c}$ is at least two times perception radius $r$, namely $r_{c} \geq 2 r$. On this condition, the researchers only need to consider the coverage problem in the sensor network, if the network is covered then it is connected [24].

Let $P_{\text {cov }}\left(x, y, s_{i}\right)$ represent the probability that any point $(x, y)$ of monitoring area $A$ is covered by sensor node $s_{i}\left(x_{i}, y_{i}\right)$ then

$$
P_{\text {cov }}\left(x, y, s_{i}\right)= \begin{cases}1, & \left(x-x_{i}\right)^{2}+\left(y-y_{i}\right)^{2} \leq r^{2} \\ 0, & \text { else }\end{cases}
$$

As long as there is a sensor node, $s_{i}$ covers the monitoring point $(x, y)$ then the monitoring point $(x, y)$ is covered by the sensor node set $S$. So the regional coverage area of sensor node set $k$ is expressed as follows:

$$
A_{\text {area }}(S)=\sum_{x=1}^{m} \sum_{y=1}^{n} P_{\operatorname{cov}}(x, y, S) \Delta x \Delta y
$$

The area of monitoring area is $A_{s}$, the working sensor node set is $S^{\prime}$, the target function of network coverage of working node set is as follows:

$$
f_{1}\left(S^{\prime}\right)=A_{\text {area }}\left(S^{\prime}\right) / A_{s}
$$

where $A_{\text {area }}\left(S^{\prime}\right)$ is the cover area of working sensor node set $S^{\prime}$.

The target function of nodes utilization rate is as follows:

$$
f_{2}\left(S^{\prime}\right)=\left|S^{\prime}\right| / N
$$

where $N$ is the total number of sensor nodes, $\left|S^{\prime}\right|$ is the number of working sensor nodes.

The aims of wireless sensor network node optimal coverage are to reduce the sensor node's utilization and to ensure maximum network coverage at the same time, this is a multi-objective optimization problem. So the multi-objective optimization coverage model of wireless sensor network is expressed as follows:

$$
\max f\left(S^{\prime}\right)=\max \left(f_{1}\left(S^{\prime}\right), 1-f_{2}\left(S^{\prime}\right)\right)
$$

\section{Improved genetic algorithm and binary ant colony algorithm}

\subsection{Genetic algorithm}

The genetic algorithm (GA) is a kind of self-adapting heuristic global search algorithm which was derived from imitating the thought of natural biological evolution. In nature, it is a cycle process made up of reproduction-crossovermutation operators. In the process of searching for the global optimum solution, GA needs neither the information of gradient nor the calculus computing, it can find out the global optimum solution or near-optimal solution in the solution space with high probability only by operating the reproduction-crossover-mutation operators; thereby, it could reduce the probability of getting into the local minimum efficiently.

Adaptive genetic algorithm (AGA) is a kind of GA that has scale reproduction and self-adaptive crossover and mutation operations. In the process of searching for the optimum parameter, AGA changes the crossover probability and mutation probability adaptively according to the different conditions of individuals in order to keep the diversity of colony and prevent the premature convergence; further, it can enhance the calculating speed and precision of the algorithm.

$$
\begin{aligned}
& P_{c}=\left\{\begin{array}{lr}
k_{1}\left(f_{\text {max }}-f^{\prime}\right) /\left(f_{\text {max }}-f_{\text {avg }}\right) & \text { if } f^{\prime}>f_{\text {avg }} \\
k_{3} & \text { if } f^{\prime}<f_{\text {avg }}
\end{array}\right. \\
& P_{m}=\left\{\begin{array}{lr}
k_{2}\left(f_{\text {max }}-f\right) /\left(f_{\text {max }}-f_{\text {avg }}\right) & \text { if } f>f_{\text {avg }} \\
k_{4} & \text { if } f<f_{\text {avg }}
\end{array}\right.
\end{aligned}
$$

where $P_{c}$ is exchanging probability, $P_{m}$ is mutation probability, $f_{\max }$ is the biggest fitness of colony, $f_{\text {avg }}$ is the average fitness of colony, $f^{\prime}$ is the bigger fitness of two strings used for crossover, $f$ is the fitness of the individual to mutate.

\subsection{Binary ant colony algorithm}

The ant colony algorithm is a kind of new simulated evolutionary algorithm. Ants will release a peculiar pheromone in its path when they are searching for food, which make other ants feel the kind of peculiarity and tend towards the direction of high density [25]. Ant colony algorithm converge the optimal path through the accumulation and update of pheromone and achieve optimization through the appropriate times of iteration. 
The binary ant colony algorithm denotes the parameters directly with binary-coding; thus, it can take up less storage space and reduce the complexity of the algorithm compared to decimal-coding. Moreover, the traversal search capability of binary-coding is better than that of decimal-coding.

Definition of the directed graph $G=(V, R)$, the vertex set

$V$ is:

$$
\left\{\left\{v_{0}\left(c_{s}\right), v_{1}\left(c_{N}^{0}\right), v_{2}\left(c_{N}^{1}\right), v_{3}\left(c_{N-1}^{0}\right), v_{4}\left(c_{N-1}^{1}\right), \cdots\right.\right.
$$

$$
\left.\left.v_{2 N-3}\left(c_{2}^{0}\right), v_{2 N-2}\left(c_{2}^{1}\right), v_{2 N-1}\left(c_{1}^{0}\right), v_{2 N}\left(c_{1}^{1}\right)\right\}\right\},
$$

where $c_{s}$ is the start vertex, $c_{j}^{0}, c_{j}^{1}$, respectively, stand for the 0 and 1 states of bit $b_{j}$ in binary-coding. For all vertex, when $j=2,3, \cdots, N$, there are only two directed arcs which point to $c_{j-1}^{0}$ and $c_{j-1}^{1}$, these two directed arcs respectively stand for the 0 and 1 states of each ant next step allowed, the $N$ is the binary encoding length, this binary ant colony network is shown in Fig. 1. Each ant travels their own path and then get the solution of the problem by integrating each ant's traversal solution [26-31].

At the start, the information quantity of each path is equal, suppose $\tau_{i, j}(0)=C$ (C is a constant), $\Delta \tau_{i, j}=0(i, j=1$, $2, \cdots, N)$. In the process of movement, the $k(k=1,2, \cdots$, $M)$ ant determines the movement direction according to the information quantity of each path, and the movable probability is

$$
p_{i, j}^{k}=\left(\tau_{i, j}^{\alpha}(t) \cdot \eta_{i, j}^{\beta}(t)\right) /\left(\sum_{s \in \text { allowed }_{k}} \tau_{i, s}^{\alpha}(t) \cdot \eta_{i, s}^{\beta}(t)\right)
$$

where $m$ is the number of ants, $p_{i, j}^{k}$ is the probability that the $k$ ant moves from the $i$ position to the $j$ position at the $t$ moment. $\alpha(\alpha \geq 0)$ is the relative importance of a moving path. $\beta(\beta \geq 0)$ is the relative importance of visibility. $\tau_{i, j}$ is the residual information amount in the $i j$ connection at the $t$ moment. $\eta_{i, j}$ is the visibility in the $i j$ connection. allowed $d_{k}=\{0,1\}$ is the next select status.

With the passage of time, the leaving information gradually disappear. $\rho(0 \leq \rho \leq 1)$ is persistence of a moving path. $1-\rho$ is the information loss degree. After $n$ moment, the ant completes one cycle. The $\tau_{i, j}$ is renewed as follows:

$$
\tau_{i, j}(t+1)=\rho \cdot \tau_{i, j}(t)+\Delta \tau_{i, j}
$$

where $\Delta \tau_{i, j}=1 / f_{\text {best }}(S), f_{\text {best }}(S)$ is the iteration-best solution or global best solution, which can enhance the optimizing ability and improve the speed of solution.

Compared with the traditional ant colony algorithm, the biggest difference of binary ant colony algorithm is the ant's path selection. The states of binary ant colony algorithm only have 0 and 1 , the ant does not need to remember those nodes which have traveled, it selects only on the basis of prior to the two path pheromones. The traverse path in the binary ant colony algorithm is shown in Fig. 2.

\subsection{Improved genetic algorithm and binary ant colony algorithm}

The improved genetic algorithm and binary ant colony algorithm introduce a genetic operator into the binary ant colony algorithm, using the crossover and mutation operations to expand the search space and enhance the global property of solutions [26, 32]. The solution is formed by the binary ant colony algorithm as an initial population of genetic algorithm then a better solution is searched by iterative evolution. Thus, it can enhance the convergence speed of algorithm, improve efficiency for solution, and avoid the local optimal and precocious defects [32].

Randomly generated initial solution executes multiple evolutionary using the reproduction-crossovermutation operators of genetic algorithm and, at the same time, using the solution obtained by genetic algorithm to initialize the information pheromones of a network. If the loop end conditions are met, then the algorithm ends; else, the researchers execute the ants' traverse and pheromone update and reserve the optimal solution, execute the reproduction-crossover-mutation operation to the obtained solution set and reserve the optimal solution, and use the optimal update pheromone and determine whether the end condition is met.

The algorithm flow is shown in Fig. 3.

Fig. 1 Binary ant colony network

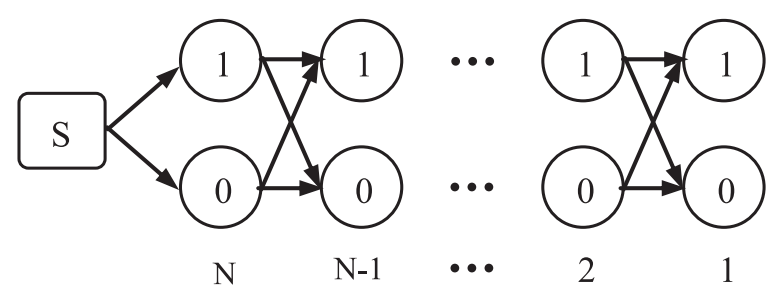




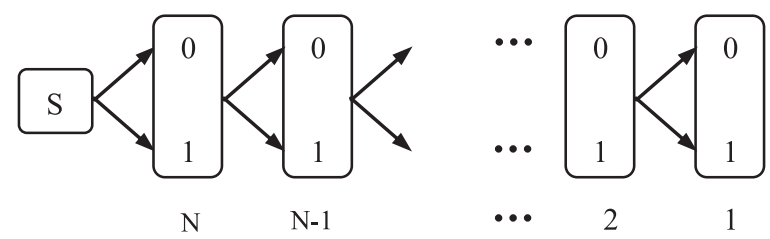

Fig. 2 Traverse path in the binary ant colony algorithm

\section{Wireless sensor networks nodes optimal coverage based on IGA-BACA}

The wireless sensor network node optimal coverage is a typical multi-objective optimization problem, this problem can be expressed as follows: existing sensor set $S=\left(s_{1}, s_{2}, \cdots, s_{i}, \cdots, s_{N}\right)$, seeking the working set $S^{\prime}$, which can obtain the maximum network coverage rate, and the minimum working node set. When the maximum network coverage rate is selected as the optimal objective, network sensor nodes with hexagon structure distributed in the monitoring area, the minimum working nodes set can be obtained [33].
From formula (5), we know that the maximum network coverage $f_{1}\left(S^{\prime}\right)$ and minimum working nodes set $f_{2}\left(S^{\prime}\right)$ are contradictory; compromising the two, the multi-objective optimization coverage model of wireless sensor network is changed into the maximum objective function $f\left(S^{\prime}\right)$.

$$
f\left(S^{\prime}\right)=\left(f_{1}\left(S^{\prime}\right) \cdot f_{1}\left(S^{\prime}\right)\right) / f_{2}\left(S^{\prime}\right)
$$

The algorithm implementation of wireless sensor network node optimal coverage based on improved genetic algorithm and binary ant colony algorithm is as follows [34-39].



Fig. 3 Flow chart of improved genetic algorithm and binary ant colony algorithm 


\subsection{Code}

It is a key problem to complete the mapping from the solution space of optimization problem to the coding space. Aiming at the random distribution nodes, the control vector (individual) $L=\left(l_{1}, l_{2}, \cdots, l_{i}, \cdots, l_{N}\right)$ of wireless sensor node is expressed in a binary coded form, which stands for the position of all sensor nodes in the target monitoring area $A$, where the $l_{i}$ value is 1 or 0 , which separately correspond to the active or sleep of the $i$ sensor node. The selection of nodes and the gene of chromosome is one to one correspondence.

\subsection{Initial solution and adaptation function}

At random initialization, ten sensor nodes randomly distribute in the target monitoring area $A$, where the nodes of $1,2,3,5,7,8,10,11,12,15$ are working nodes and then the corresponding individual is $\left\{\begin{array}{lllllll}1 & 1 & 1 & 0 & 1 & 0 & 1\end{array}\right.$

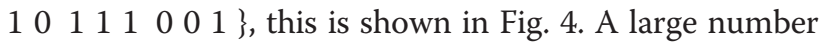
of individuals will be generated in the initialization phase, which is called a colony.

Use the formula (10) as the adaptation function, the higher is the adaptation function value, the higher is the selected probability of individual.

\subsection{Operation operator}

The operation operator of genetic algorithm and binary ant colony algorithm is composed of four operators that is reproduction operator, crossover operator, mutation operator, and pheromone update operator.

The reproduction operator reproduces the individuals to the new colony according to the probability in proportion as their adaptive value. After reproduction, the preponderant individuals are preserved and the inferior individuals are weeded out, and the average fitness degree of the colony is increased, but the variety of colony is lost at the same time. The action of reproducing an operator is to realize the principle of winner priority for preserving predominance and natural selection and making the colony converge on the optimum solution.
The crossover operator first selects two individuals stochastically according to the certain adaptive exchanging probability $P_{c}$ then it can produce two new individuals by exchanging parts of chromogene stochastically. The twopoint crossover is adopted in this paper, randomly generating two crossover points is shown in Fig. 5. The genetic algorithm can generate filial generation colony which have higher average fitness and better individuals through the reproduction and crossover operators and can make the evolutionary process proceed to the optimum solution.

The mutation operator changes several bits of the chromosome string stochastically with a small probability $P_{m}$, namely turning 0 to 1 and 1 to 0 . The mutation operator is very important to recoup the loss of colony diversity.

The pheromone update operator $\left(T_{g}\right)$ is a mapping of pheromone update for optimal individual which is selected from an ant sequence. According to the MaxMin rule, pheromone only be released in the path of optimal solution ant traverse, namely $\Delta \tau_{i, j}=1 / f_{\text {best }}(S)$, $f_{\text {best }}(S)$ is the iteration-best solution or global best solution. The probability of pheromone update operator is shown as follows:

$$
P\left\{T_{g}=x_{i}\right\}=f\left(x_{i}\right) / \sum_{k=1}^{N} f\left(x_{k}\right)
$$

where, $N$ is the number of individuals.

\subsection{Ants traverse}

When an ant travels, the path selection of different ants is mutual independence. Starting from the nest, every ant performs path selection with the movable probability formula (8) and according to Fig. 2; because of the binary-coding, the ants do not possess memory function, it selects only on the basis of prior to the two path pheromones. The path that has higher density pheromone will have higher selection probability. When an ant completes the path selection in an ant sequence, it arrives at the food source.

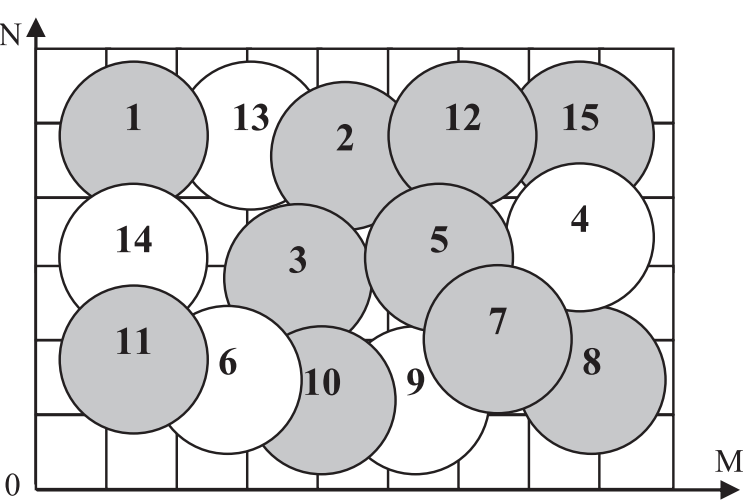

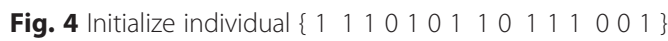




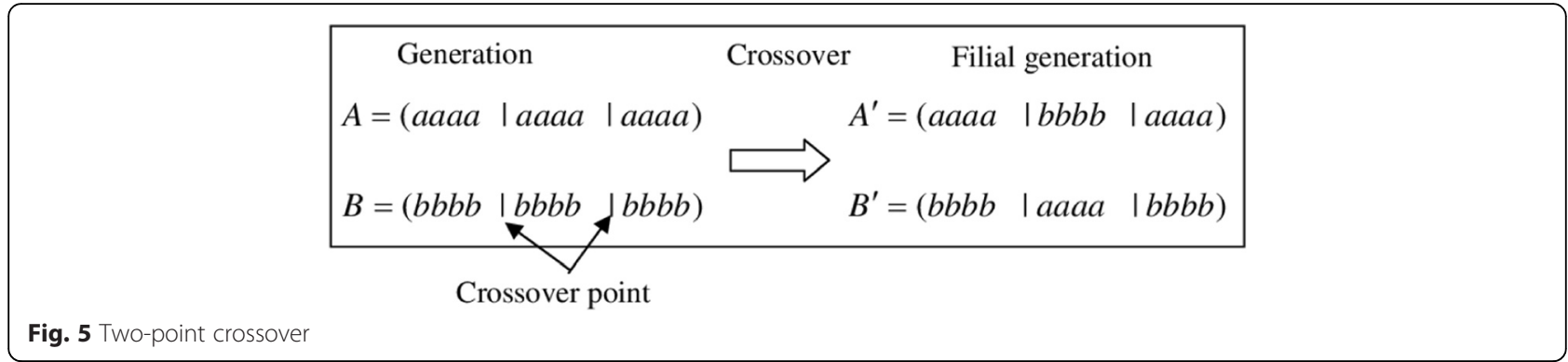

\section{Simulation experiment}

The set of the monitoring area $A$ is $100 m \times 100 m$, the perception radius $r$ of sensor nodes is $10 \mathrm{~m}$. When the network sensor nodes with hexagon structure distributed in the monitoring area and the distance $d$ between nodes are $d=\sqrt{3} \cdot r$, the coverage of target monitoring area $A$ can achieve optimal. From this, we know that 45 nodes with a radius of 10 can completely cover the monitoring area $A$, this is shown in Fig. 6.

We write the corresponding algorithm program using $\mathrm{C}++$. The improved genetic algorithm and binary ant colony algorithm adopt two-point crossover, scale reproduction, and self-adaptive crossover and mutation operations. Population size is 60 . There was random distribution of 100 sensor nodes in monitoring area $A$, the perception radius of sensor nodes is $10 \mathrm{~m}$ then every individual is composed of 100 binary bits. Algorithm iterative number is 200 . Figure 7 is the 100 nodes in the area $A$ random distribution. Although the nodes can cover the target region, but there are a lot of redundant nodes.
Figure $8 \mathrm{a}-\mathrm{d}$ shows the working nodes set coverage using the improved genetic algorithm and binary ant colony algorithm evolution 50, 100,150, 200 generations.

From Fig. 8a-d, we can see that the algorithm search towards the global optimal solution direction. When the iterative number is 200 , the sensor nodes is near to the optimal distribution, using less number of sensor nodes, obtaining more than $97 \%$ coverage.

In order to evaluate the performance of IGA-BACA algorithm, in the other parameters unchanged, we evaluate its performance using different random deployment scenarios. In different sensor node density experiments, the number of sensor nodes deployed is, respectively, $100,120,150,180$, and 200. From the network coverage rate and the convergence of the algorithm, the improved genetic algorithm and binary ant colony algorithm are compared with ACA algorithm and GA algorithm. The experimental results are the average of 30 experiments. Figure 9 is a network coverage rate comparison chart of different sensor nodes.

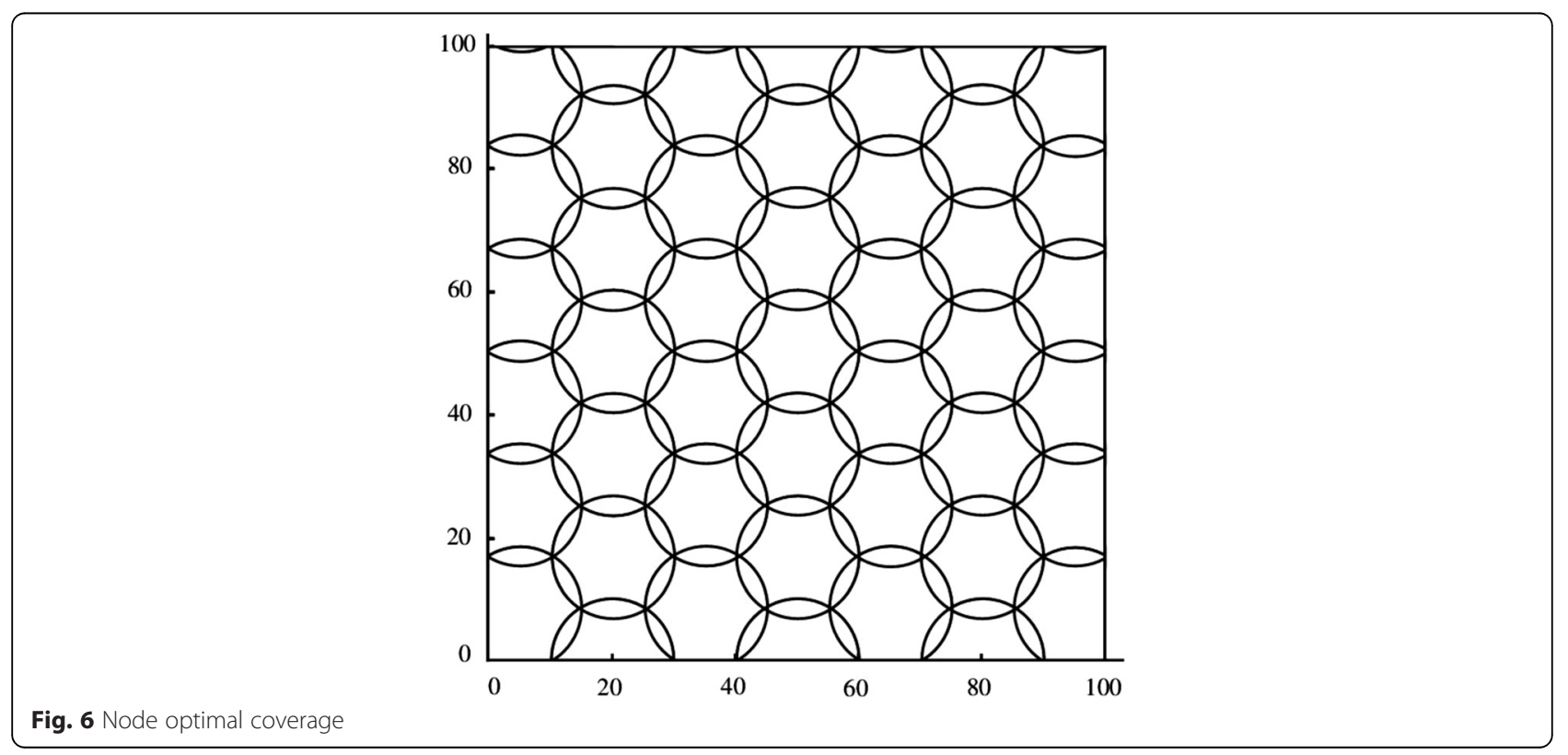




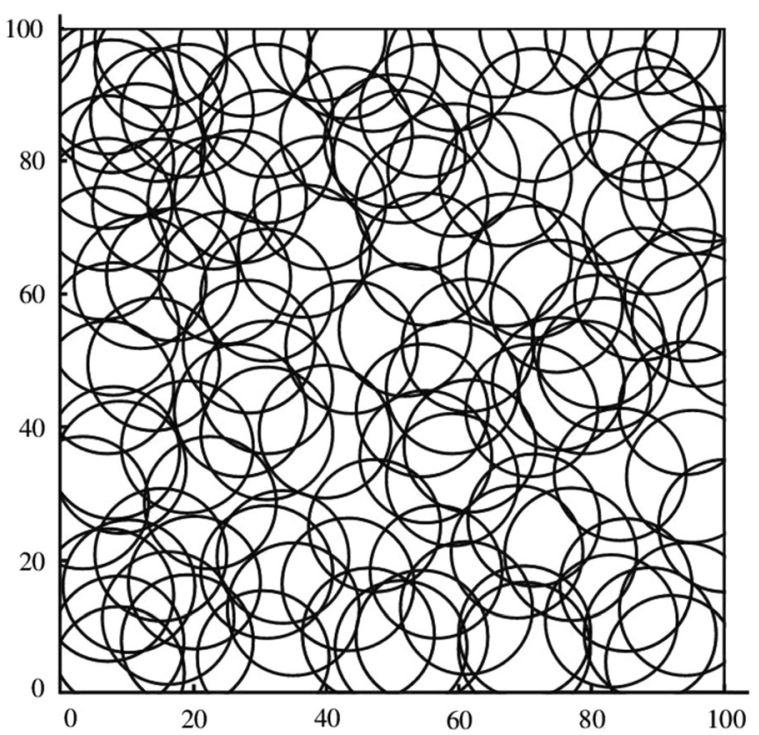

Fig. 7 Random distribution 100 nodes in the area $A$

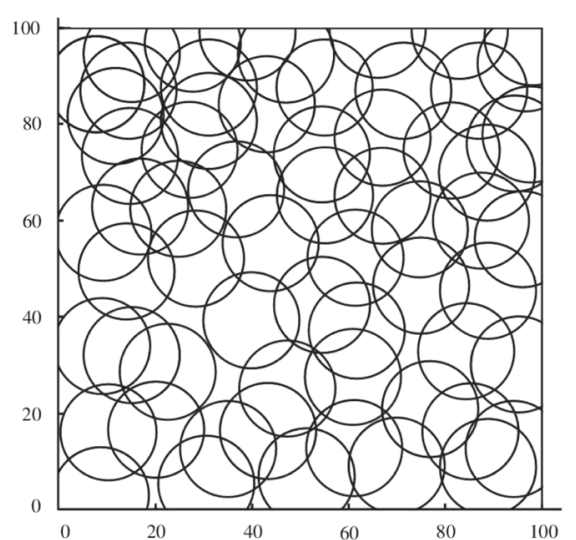

(a) 50 generation

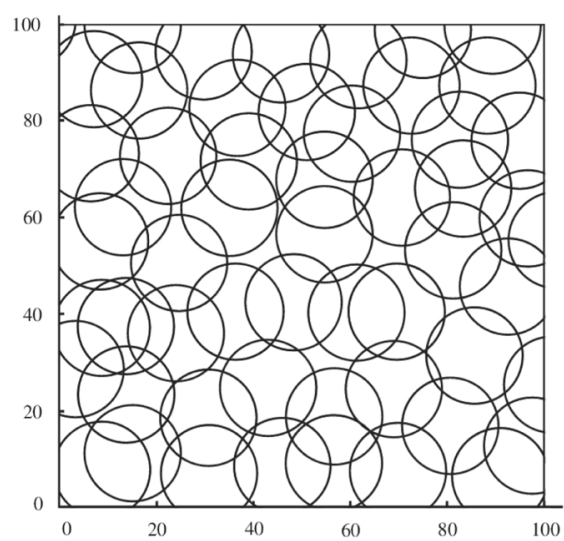

(b) 100 generation

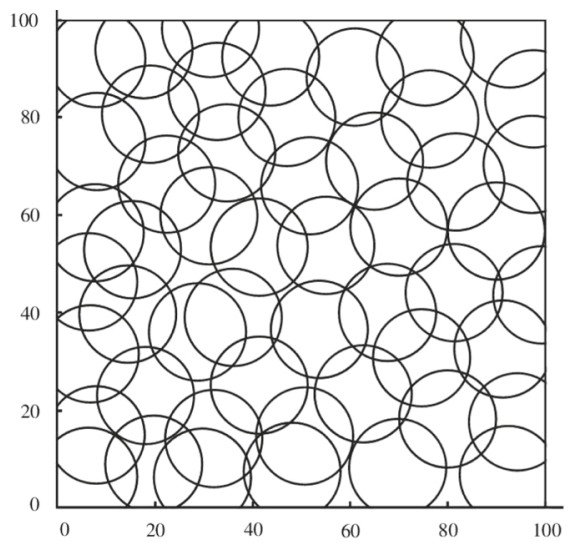

(c) 150 generation

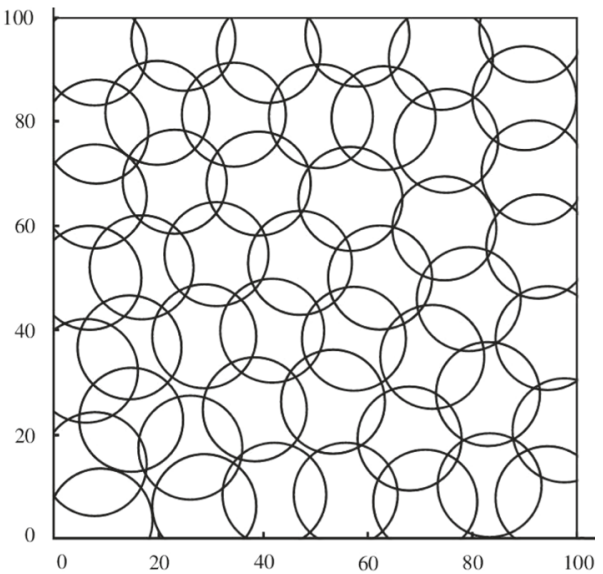

(d) 200 generation

Fig. 8 Working set node coverage by IGA-BACA evolution. a 50 generations, b 100 generations, c 150 generations, d 200 generations 


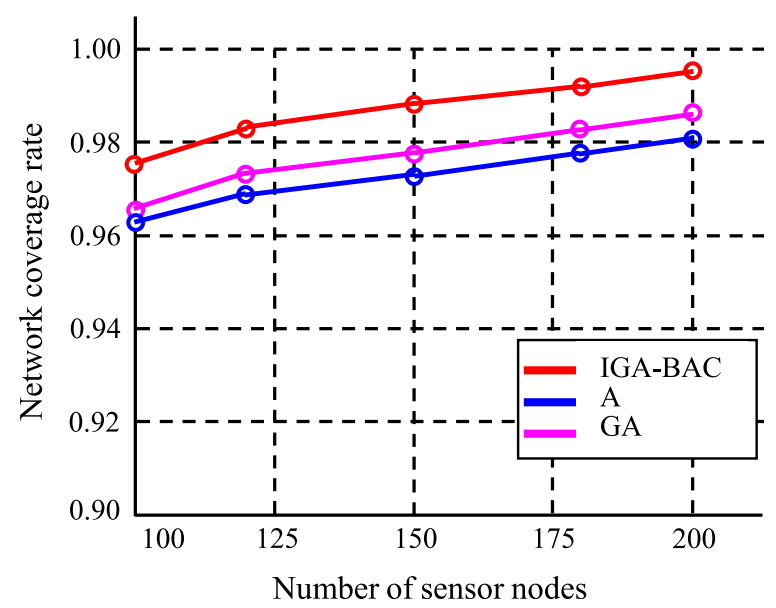

Fig. 9 Network coverage rate comparison chart

From Fig. 9, we can see that the coverage quality increases along with the increase of sensor node density. In the same iteration, coverage quality of IGA-BACA algorithm is higher than those of GA algorithm and ACA algorithm. After 200 generations of evolution, the number of network sensor node is 100, the network coverage rate of IGA-BACA algorithm is $97.5 \%$, the network coverage rate of GA algorithm is $96.2 \%$, and the network coverage rate of ACA algorithm is $96.5 \%$. The number of network sensor node is 150 , the network coverage rate of IGA-BACA algorithm is $98.7 \%$, the network coverage rate of GA algorithm is $97.3 \%$, and the network coverage rate of ACA algorithm is $97.7 \%$. The number of network sensor node is 200, the network coverage rate of IGA-BACA algorithm is $99.5 \%$, the network coverage rate of GA algorithm is $98.1 \%$, and the network coverage rate of ACA algorithm is $98.6 \%$. These results show that the IGA-BACA algorithm is better than the ACA algorithm and GA algorithm in wireless sensor network coverage quality improvement.

Figure 10 is the convergence comparison chart of IGA-BACA algorithm, GA algorithm, and ACA algorithm in 200 generations of the evolutionary process. Figure 10a is the convergence comparison chart in the 100 sensor nodes deployed, and the Fig. 10b is the convergence comparison chart in the 200 sensor nodes deployed. Figure 10 shows the convergence of the three algorithms. From Fig. 10a, we can see that this figure clearly shows the convergence of the three algorithms' performance. The IGA-BACA algorithm, GA algorithm, and $\mathrm{ACA}$ algorithm can converge, and the convergence of IGA-BACA algorithm is better than that of ACA algorithm and GA algorithm, the convergence of ACA algorithm is better than that of GA algorithm. From Fig. 10b, we can get the same conclusion, for the three algorithms, and the convergence of IGA-BACA is the best, followed by ACA, finally GA. So the performance of IGA-BACA is better than the GA algorithm and ACA algorithm under the same parameters.

In order to further illustrate the validity of the IGABACA, we adopt IGA-BACA and GA to realize the optimal coverage of wireless sensor network node in the same conditions. In the 100 sensor nodes deployed, the simulation results by IGA-BACA and GA are shown in Table 1 .

From Table 1, we can see that the network coverage rate of IGA-BACA is greater than that of GA in the same evolution generation condition. Moreover, the working set node number of IGA-BACA is smaller than that of GA in the same evolution generation condition. We also can see that along with the evolution generation increase, the network coverage rate is gradually approaching the optimal solution; moreover, the working set sensor node number is gradually reduced, this will reduce energy loss of a wireless sensor network and prolong the network lifetime. From the CPU time, we can see the IGA-BACA algorithm to optimize the speed faster than the GA algorithm.

All of these show that the IGA-BACA is superior to the GA and ACA at the convergence of the optimal solution with less working nodes and high network coverage rate.

\section{Conclusions}

The coverage and energy consumption are the key problems of wireless sensor network research because the sensor node energy cannot regenerate or supply. This paper analyzes the characteristics of wireless sensor network limited node energy. With the network coverage and the number of working set nodes as the optimization object, research on the working node set selection optimization problem of nodes explore the high-density deployment in wireless sensor network.

The wireless sensor network node optimal coverage based on improved genetic algorithm and binary ant colony 


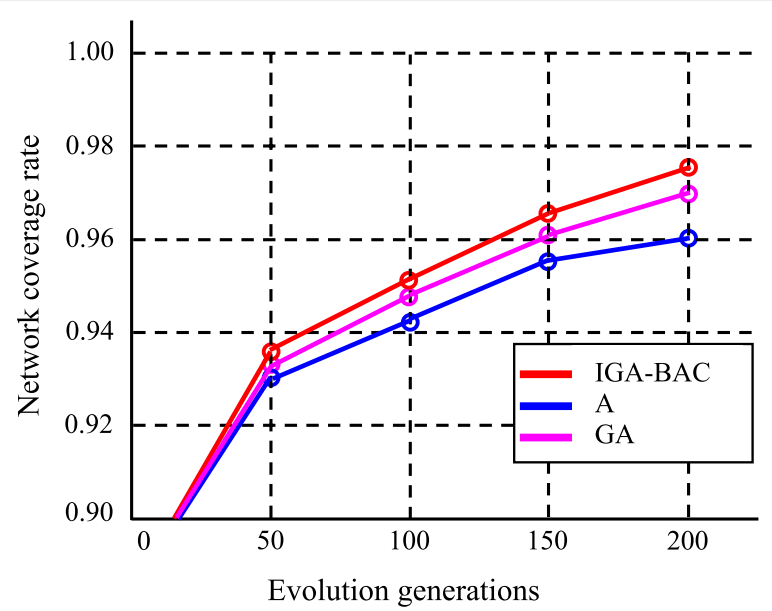

(a) The convergence of 100 sensor nodes

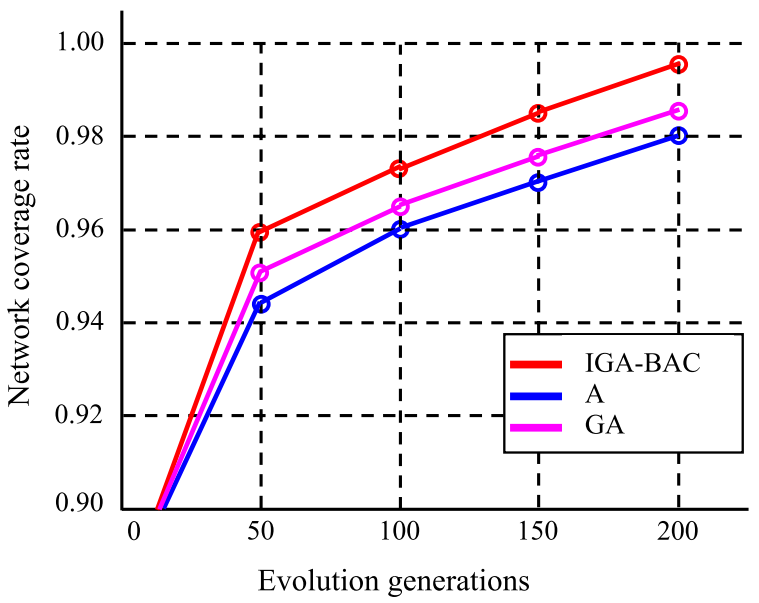

(b) The convergence of 200 sensor nodes

Fig. 10 The algorithm convergence comparison chart. a The convergence of 100 sensor nodes. $\mathbf{b}$ The convergence of 200 sensor nodes

algorithm is proposed and applied to node optimal coverage. The aim at the disadvantages of the genetic algorithm and ant colony algorithm is introduce binary ant colony algorithm and combine it with genetic algorithm to form improved genetic algorithm and binary ant colony algorithm which has higher accuracy and faster convergence speed. Considering the relationship between network coverage and the number of working set nodes, the complex multi- objective problem is simplified into a single-objective fitness function in this paper, which can balance network coverage rate and energy consumption of the system.

The simulation results show that the improved genetic algorithm and binary ant colony algorithm can achieve the optimal working set with less working nodes and high network coverage rate, effectively reducing energy loss of a wireless sensor network and prolonging the network lifetime.

Table 1 Simulation results by IGA-BACA and GA

\begin{tabular}{|c|c|c|c|c|c|c|}
\hline \multirow{2}{*}{$\begin{array}{l}\text { Evolution } \\
\text { generations }\end{array}$} & \multicolumn{3}{|l|}{ IGA-BACA } & \multicolumn{3}{|l|}{$\mathrm{GA}$} \\
\hline & $\begin{array}{l}\text { Network coverage } \\
\text { rate }(\%)\end{array}$ & $\begin{array}{l}\text { Working set } \\
\text { node number }\end{array}$ & CPU-time $(\mathrm{s})$ & $\begin{array}{l}\text { Network coverage } \\
\text { rate }(\%)\end{array}$ & $\begin{array}{l}\text { Working set } \\
\text { node number }\end{array}$ & CPU-time (s) \\
\hline- & 100 & 45 & - & 100 & 45 & - \\
\hline 50 & 93 & 63 & 11 & 93.1 & 65 & 13 \\
\hline 100 & 95.2 & 56 & 21 & 94.3 & 59 & 25 \\
\hline 150 & 96.5 & 51 & 31 & 95.6 & 53 & 37 \\
\hline 200 & 97.5 & 48 & 42 & 96.2 & 50 & 49 \\
\hline
\end{tabular}




\section{Competing interests}

The authors declare that they have no competing interests.

\section{Acknowledgements}

This work is supported in part by the National Natural Science Foundation of China under Grant No. 61271370, the National High Technology Research and Development Program of China (863 Program) under Grant No. 2013AA013202, and Funding Project for Academic Human Resources Development in Beijing Union University No. 11101501105.

Received: 28 December 2015 Accepted: 4 April 2016

Published online: 14 April 2016

\section{References}

1. IF Akyildiz, W Su, Y Sankarasubramaniam, E Cayirci, Wireless sensor networks: a survey. Comput. Netw. 38, 393-422 (2002)

2. I.T. Almalkawi, Guerrero Zapata, M., Al-Karaki, J.N. A cross-layer-based clustered multipath routing with QoS-aware scheduling for wireless multimedia sensor networks. Int. J. Distributed Sensor Net. 2012, 1-11 (2012)

3. J Yang, C Zhang, X Li, Y Huang, S Fu, MF Acevedo, Integration of wireless sensor networks in environmental monitoring cyber infrastructure. Wirel. Netw 16(4), 1091-1108 (2010)

4. H Chi-Fu, T Yu-Chee, The coverage problem in a wireless sensor network. Mobile Network Appl. 10(4), 519-528 (2005)

5. J Yick, B Mukherjee, D Ghosal, Wireless sensor network survey. Comput. Netw. 52, 2292-2330 (2008)

6. H Alemdar, C Ersoy, Wireless sensor networks for healthcare: a survey. Comput. Netw. 54, 2688-2710 (2010)

7. Y LIANG, P Zeng, $\mathrm{H}$ Yu, Energy adaptive cluster-head selection for wireless sensor networks. Inf. Control. 35, 141-146 (2006)

8. M Cardei, D Ding-Zhu, Improving wireless sensor network lifetime through power aware organization. Wirel. Netw 11, 333-340 (2005)

9. X. R. Wang, G. L. Xing, Y. F. Zhang, C. Y. Lu, R. Pless, C. D. Gill. Integrated coverage and connectivity configuration in wireless sensor networks. Proceedings of the 1st ACM Conference on Embedded Networked Sensor Systems (SenSys'03), Los Angeles, CA,2003.

10. G Joon-Min, H Youn-Hee, A target coverage scheduling scheme based on genetic algorithms in directional sensor networks. Sensors, Sensor 11, 1888-1906 (2011). doi:10.3390/s11020188

11. J Jia, J Chen, J Chang, L Zhao, G Wang, Optimal coverage scheme based on genetic algorithm in wireless sensor networks. Control Decis. 1(22), 1289-1292 (2007)

12. MM Islam, M Ahasanuzzaman, MA Razzaque, MM Hassan, A Alelaiwi, Y Xiang, Target coverage through distributed clustering in directional sensor networks. EURASIP J. Wireless Commun. Netw. 3, 1-18 (2015)

13. $X\llcorner u o, Z X u, J Y u, X$ Chen, Building association link network for semantic link on web resources. IEEE Trans. Autom. Sci. Eng. 8(3), 482-494 (2011)

14. Z. Xu et al. Crowdsourcing based social media data analysis of urban emergency events. Multimed Tools Appl. doi:10.1007/s1 1042-015-2731-1.

15. Z. Xu et al. Crowdsourcing based description of urban emergency events using social media big data. IEEE Transactions on Cloud Computing. doi:10. 1109/TCC.2016.2517638.

16. Z Xu et al., Participatory sensing based semantic and spatial analysis of urban emergency events using mobile social media. EURASIP J. Wirel. Commun. Netw. 44, 2016 (2016)

17. Z. Xu et al. Building knowledge base of urban emergency events based on crowdsourcing of social media. Concurr. Comput. doi: 10.1002/cpe.3780.

18. HM Ammari, On the problem of $\mathrm{k}$-coverage in mission-oriented mobile wireless sensor networks. Comput. Netw. 56(7), 1935-1950 (2012)

19. E. Lattanzi, A. Bogliolo, VirtualSense. A java-based open platform for ultra-lowpower wireless sensor nodes. Int. J. Distributed Sensor Netw. 2012, 1-16 (2012)

20. C Wenping, C Si, L Deying, Minimum-delay POls coverage in mobile wireless sensor networks. EURASIP J. Wireless Commun. Netw 2013, 1-12 (2013)

21. Y Sun, Y Sun, Z Fang, Connectivity and coverage of wireless sensor networks. J. Tianjin University 38(1), 14-17 (2005)

22. S. Meguerdichian, F. Koushanfar, M. Potkonjak et al, Coverage problem in wireless ad-hoc sensor networks. IEEE INFOCOM, vol 3 (IEEE Press, IEEE, Anchorage, 2001) 1380-1387.

23. $M$ Liu, J Cao, $Y$ Zheng, $L$ Chen, $L$ Xie, Analysis for multi-coverage problem in wireless sensor networks. J. Softw. 18, 127-136 (2007)
24. H. Do. Research on the coverage and connectivity algorithm of wireless sensor network, Master degree thesis, Hunan University, (2007) p. 41-45.

25. Y Sun, JW Tian, WSN path optimization based on fusion of improved ant colony algorithm and genetic algorithm. J. Comput. Info. Syst. 6, 1591-1599 (2010)

26. W Xiong, P Wei, Binary ant colony evolutionary algorithm. Acta Automat. Sin. 33, 259-264 (2007)

27. B Yan, W Xiong, M Cheng, Q Ye, Multi-population binary ant-colony algorithm with congestion control strategy. Control Theory \& Applications 26(4), 387-394 (2009)

28. Q Qian, M Cheng, W Xiong, M Zhou, Reviews of binary ant colony optimization. Appl. Res. Computers 29(4), 1211-1215 (2012)

29. C Yan, W Xiong, Y Zhang, Binary ant colony optimization based on traffic flow control. J. Syst. Simulation 19(10), 2346-2350 (2007)

30. W Xiong, P Wei, J Zhao, Binary ant colony algorithm with signal transfer. Pattern Recognit. Artif. Intell. 20(1), 15-20 (2007)

31. G Hu, W Xiong, X Zhang, J Yuan, Binary ant colony algorithm with controllable search bias. Contr. Theor. Appl. 28(8), 1071-1080 (2011)

32. J Pan, X Wang, Y Chen, Improved ant colony algorithm for mobile robot path planning. J. China University of Mining \& Technology 41(1), 108-113 (2012)

33. Z Honghai, JC Hou, Maintaining sensing coverage and connectivity in large sensor networks. Ad- hoc and Sensor Wirel. Netw 1, 89-124 (2005)

34. Y Zeng, J Cao, J Hong, S Zhang, L Xie, Secure localization and location verification in wireless sensor networks: a survey. J. Supercomput. 64(3), 685-701 (2013)

35. WSH Siu, C-K Chan, HCB Chan, Green cargo routing using genetic algorithms. Lect. Notes Eng. Comput. Sci. 1, 170-175 (2012)

36. A Chen, S Kumar, TH Lai, Local barrier coverage in wireless sensor networks. IEEE Trans. Mob. Comput. 9(4), 491-504 (2010)

37. X. Yu, J. Zhang, J. Fan, and T. Zhang. A faster convergence artificial bee colony algorithm in sensor deployment for wireless sensor networks. Int. J. Distributed Sensor Netw. 2013, 1-9 (2013).

38. M Koç, I Korpeoglu, Coordinated movement of multiple mobile sinks in a wireless sensor network for improved lifetime. EURASIP J. Wireless Commun. Netw. 2015, 1-16 (2015)

39. S Jin-Taek, L Heung-No, 4-ary network coding for two nodes in cooperative wireless networks: exact outage probability and coverage expansion. EURASIP J. Wireless Commun. Netw. 2012, 1-12 (2012)

\section{Submit your manuscript to a SpringerOpen ${ }^{\mathcal{O}}$ journal and benefit from:}

- Convenient online submission

- Rigorous peer review

- Immediate publication on acceptance

- Open access: articles freely available online

- High visibility within the field

- Retaining the copyright to your article

Submit your next manuscript at springeropen.com 